\title{
Design of a wireless active sensing unit for localized structural health monitoring
}

\author{
Jerome Peter Lynch*,† \\ Department of Civil and Environmental Engineering, University of Michigan, U.S.A.
}

\begin{abstract}
SUMMARY
The recent years have witnessed an increasing interest in using wireless structural monitoring as a low-cost alternative to tethered monitoring systems. Previous work considered wireless sensors strictly as passive elements in the monitoring system, responsible only for collection of response measurements. This paper explores expansion of the wireless structural monitoring paradigm by including actuation capabilities in the design of a wireless active sensing unit. To validate the performance of the prototype unit in structural health monitoring applications, an aluminum plate monitored by piezoelectric active sensors is used. Piezoelectric actuators mounted to the surface of the plate are commanded by the wireless active sensing unit to excite and record the element. System identification models are then used to model the linear relationship between the input excitation and the corresponding plate response. A novel damage detection methodology is proposed that uses the characteristic equation roots obtained from an autoregressive with exogenous input time-series model. Complex roots (poles) of the model's characteristic equation are sensitive to structural damage causing a change in their location on the complex plane. Using the mean value of pole clusters, the migration of model poles are shown to be well correlated to the severity of crack damage intentionally introduced in the plate. Copyright (C) 2005 John Wiley \& Sons, Ltd.
\end{abstract}

KEY WORDS: wireless sensors; active sensing; Lamb waves; structural health monitoring; damage detection

\section{INTRODUCTION}

Civil structures are expensive assets that contribute to the long-term prosperity of society. In recent years, many critical civil structures have been deemed to be in deteriorating states as a result of poor long-term maintenance or excessive loading. For example, a significant portion of the national inventory of highway bridges in the United States are rated structurally deficient because they carry traffic loads that exceed those anticipated when the bridges were initially

\footnotetext{
*Correspondence to: Professor J. P. Lynch, Department of Civil and Environmental Engineering, University of Michigan, 2328 G. G. Brown Building, Ann Arbor, MI 48109-2126. U.S.A.

${ }^{\dagger}$ E-mail: jerlynch@umich.edu

Contract/grant sponsor: US National Science Foundation; contract/grant numbers: CMS-9988909, CMS-0421180 Contract/grant sponsor: Los Alamos National Laboratory; contract/grant number: 75067-001-03

Contract/grant sponsor: University of Michigan
}

Copyright (C) 2005 John Wiley \& Sons, Ltd.

Received 24 August 2004

Revised 9 November 2004 Accepted 10 December 2004 
designed [1]. Furthermore, civil structures located in regions of high seismic activity can sustain major damage during strong earthquakes. Recognizing the pressing nature of these safety issues, the structural engineering community routinely prescribes inspection of civil structures to search for signs of deterioration and damage. Inspection methods that are available include visual inspection and nondestructive evaluation (NDE). While many of these inspection methods have played major roles in assessing structural health, they suffer from some limitations. For instance, visual inspections can only assess structural health from external signs of structural distress and are dependent on the judgment of the inspector; NDE techniques, including ultrasonic acoustic inspection, are local approaches to structural inspection that can be laborintensive, time-consuming and expensive.

An alternative to visual and NDE inspection is the use of permanent structural monitoring systems that record the response of civil structures to live loads. Structural monitoring systems employ a variety of sensors that communicate measurements via coaxial wires to centralized data repositories, where data is recorded for future engineering analysis. In particular, in areas where earthquakes pose a serious risk to civil structures, structural monitoring systems are often mandated by local law for structures of sufficient size [2]. The benefits gained by continuously monitoring civil structures are many: repositories of structural response data can be used to obtain better understanding of structural performance, to assess the effectiveness of current design methodologies and to identify the existence of structural damage. When the monitoring system is able to autonomously interrogate sensor measurements for indications of structural damage, the system is known as a structural health monitoring system. The scope (global versus local) of the damage detection method is strongly influenced by the monitoring system's underlying sensor topography. Many of the damage detection algorithms proposed by the structural engineering community consider changes in global structural vibration characteristics as indicators of damage [3]. The impetus for using global vibration characteristics can be attributed to monitoring systems employing low sensor densities intended to only capture global structural behavior. Low sensor densities are a result of the high costs of installing cable-based monitoring systems, often in excess of $\$ 5000$ per sensor [4]. The onset of structural damage in civil structures is a local phenomenon that is poorly manifested in global vibration characteristics. Furthermore, monitoring systems with low sensor densities capture only loworder system modes that also exhibit sensitivity to the environmental and operational variability common of civil structures. Increases in the density of sensors installed in large-scale civil structures is needed to better capture local structural behavior and high-order response modes so that damage detection methods will be better able to identify the initiation of structural damage.

With electronic and computing technologies declining in cost while improving at rates espoused by Moore's law [5], a reevaluation of the design of structural monitoring systems is warranted. In particular, modern technologies developed in related engineering disciplines can be adopted to broaden the capabilities of current structural monitoring systems while reducing their installation costs. In lieu of extensive cabling, Straser and Kiremidjian [6] first proposed the integration of wireless communications with sensors to reduce the cost of structural monitoring systems. Lynch [7] has extended on Straser and Kiremidjian's seminal work by enhancing the functionality of wireless sensors by integrating embedded microcontrollers with them. With computational power coupled with sensors, engineering algorithms can be embedded in the computational cores of wireless sensors for real-time execution. Lynch et al. [8] recently illustrated the utility of sensor-based data interrogation by embedding damage detection 
algorithms in a wireless sensing unit. Wireless sensors that autonomously process measurement data for damage prognosis can serve as building blocks of future structural health monitoring systems. To validate wireless monitoring of real civil structures, a structural monitoring system consisting only of wireless sensing unit prototypes was successfully used to monitor the Alamosa Canyon Bridge in New Mexico during forced vibration testing [7]. Aside from academic prototypes explicitly designed for structural monitoring, a number of generic commercial wireless sensor systems have been used by researchers during laboratory and field testing of structures $[9,10]$. As the field of wireless sensors rapidly evolves, costs will continue to decline and greater nodal densities will be employed.

The sensors used in permanent monitoring systems are passive devices that only record the behavior of the structure during ambient and forced vibrations. Recently, active sensors have received attention as a promising monitoring technology that could improve a structural health monitoring system's ability to quantify structural damage. Active sensors are sensors with an ability to initiate vibrations in a structure and to record the corresponding structural response to those vibrations. An attractive feature of active sensing is the ability to repeatedly apply the same excitation on the structure - a convenience not afforded by ambient or seismic loads. Active sensing encompasses many familiar NDE monitoring tools including impact echo and ultrasonic wave testing. The growing interest in the active sensing field lies in the increased availability and reduced cost of piezoelectric materials. A piezoelectric material expands when a voltage potential is applied; conversely, such materials will generated an internal electric potential when strained. This symmetrical mechanicalelectrical property of piezoelectric materials has motivated their use as both strain sensors and actuators. With respect to damage prognosis, piezoelectric pads are widely used as conformable surface mounted actuators for imparting Lamb waves into structural plates. Many researchers have correlated the time-of-arrival and attenuation properties of Lamb waves with crack and delamination damage in plates [11-14]. Others have illustrated the sensitivity of a piezoelectric pad's electrical impedance to damage when the structural damage is in the vicinity of the mounted pad [15].

To take advantage of active sensing technologies for monitoring the long-term health of civil structures, the wireless sensing paradigm can be further extended to include actuation capabilities. In this study, a convergence of wireless communications, mobile computing, sensors and actuators is proposed for the design a wireless active sensing unit capable of interacting with the structure in which it is installed. With the capability to actuate, sense and compute, the wireless active sensing unit is a self-contained intelligent agent ready to play ever greater roles in structural health monitoring applications. While any actuator type can be considered for use with the proposed wireless active sensing unit, piezoelectric actuation will be used for illustrative purposes in this study. To validate the performance of the proposed wireless active sensing unit design, an aluminum plate with two piezoelectric elements epoxy mounted to its surface will be studied. The wireless active sensing unit will command one piezoelectric pad to emit broad-band surface acoustic waves, while the sensing interface simultaneously records attenuated waves received at a second pad. Input-output response data of the aluminum plate fully characterizes the linear behavior of the structural element. As such, time-series system identification models formed from the data can be used to identify the existence of crack damage in the plate. In particular, the roots of an autoregressive model's characteristic equation will be shown to exhibit sensitivity to damage. The paper will conclude with a discussion of the pertinent research findings and suggest future research directions. 


\section{HARDWARE DESIGN OF THE WIRELESS ACTIVE SENSING UNIT}

Herein proposed, is the design of a wireless active sensing unit capable of: (1) commanding active sensors and actuators; (2) collecting structural response measurements from sensors; (3) storing and interrogating measurement data collected; and (4) communicating data to a wireless sensing network comprised of other wireless sensors. To accomplish these tasks, the architectural design of the wireless active sensing unit can be delineated by four functional modules: the computational core, the sensing interface, the actuation interface and the wireless communication channel. Hardware components will be selected based on their ability to satisfy the performance requirements of the wireless active sensing unit. Unlike previous wireless sensing unit designs, integration of an actuation interface that can command active sensors will place imposing performance demands on the unit design. For example, Lamb wave generation in structural elements using piezoelectric pads will require the actuation and sensing interfaces to operate at high sample rates in excess of $1 \mathrm{kHz}$. The performance goals of the sensing and actuation interfaces represent a significant challenge of the proposed unit design; as such, other design issues, such as power consumption, will not be a primary focus in this study. To keep the total cost of the wireless active sensing unit prototype low, an off-the-shelf design strategy will be employed with only commercial hardware components considered. Figure 1 is an architectural overview of the wireless active sensing unit design with all the electrical components delineated.

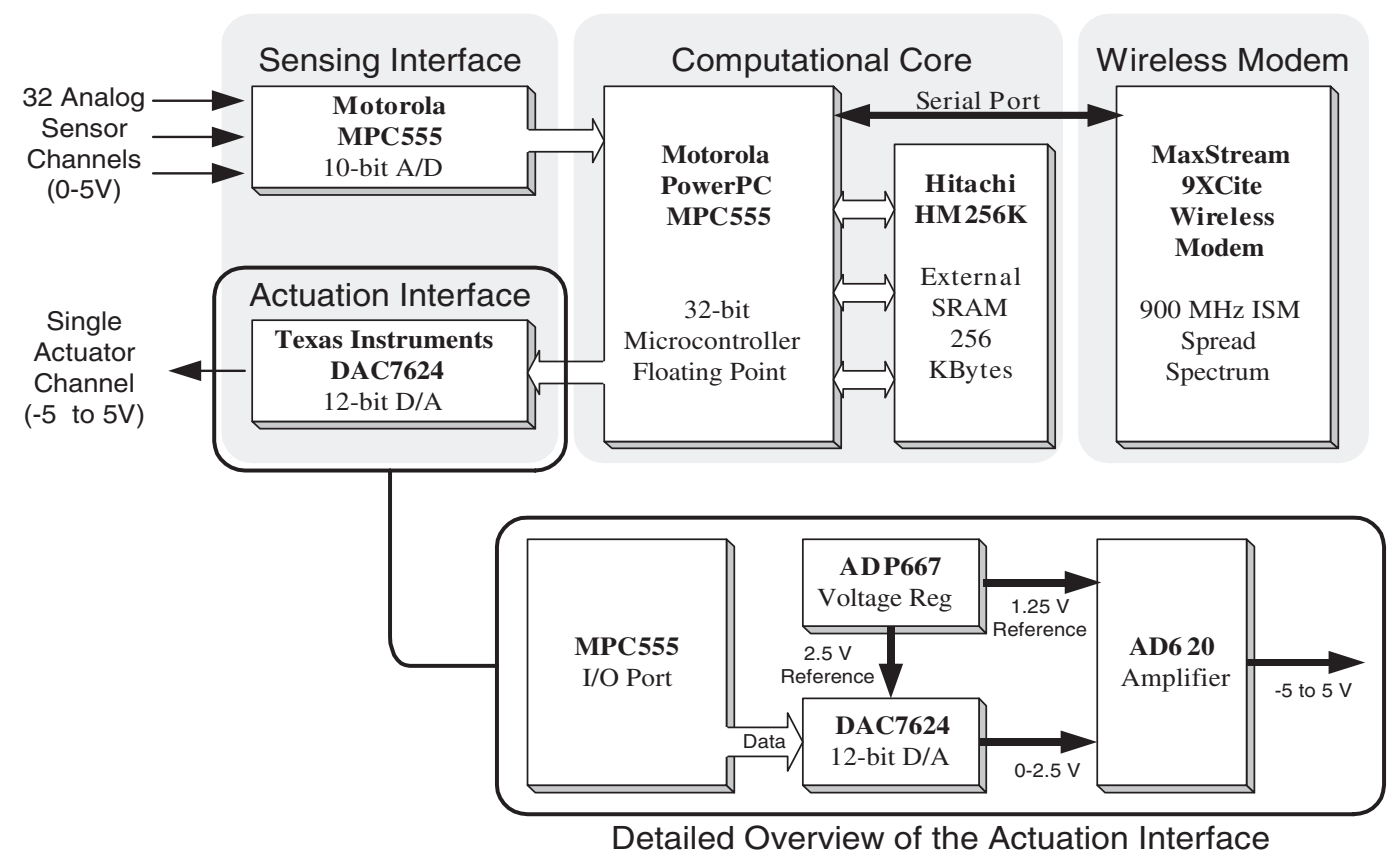

Figure 1. Proposed hardware design of a wireless active sensing unit for health monitoring of civil structures. 
At the center of the wireless active sensing unit design is the computational core. Similar to previously proposed wireless sensing unit designs, the 32-bit Motorola MPC555 PowerPC microcontroller is selected as the core's primary hardware component [7]. Selection of the MPC555 is motivated by its high-speed clock frequency $(40 \mathrm{MHz})$ and its sophisticated internal arithmetic and logic unit capable of executing floating-point calculations in hardware. High processor speeds are necessary to operate high-frequency piezoelectric elements envisioned for use with the wireless active sensing unit. Internally integrated with this fast processor is 448 kbytes of read-only memory (ROM) where firmware will be stored for unit operation and embedded data processing. Since only 26 kbytes of internal random-access-memory (RAM) is included in the MPC555, 512 kbytes of external static RAM (SRAM) is added to the unit design for data storage. The read and write operations to the external memory take two clock cycles to complete and are slower than those to internal memory that take one cycle. Therefore, for applications demanding high sample rates, data collected from the sensing interface will be written first to internal memory. After the data has been collected and real-time constraints are no longer imposing, the measurement data will be transferred automatically from internal to external memory.

The 10-bit analog-to-digital converter (ADC) that is included with the MPC555 will be used as the sole sensing interface of the wireless active sensing unit. The sensing interface can accommodate the collection of structural response data from analog sensors connected to the 32 simultaneous sensing channels readily available. A particularly convenient feature of the onchip ADC is its maximum sample rate of $100 \mathrm{kHz}$. A high sample rate is useful when using the wireless active sensing unit for recording the response of structural plates to Lamb waves. To ensure the real-time demands of the data acquisition tasks are met and that data is read from the ADC on a precise timing schedule, interrupt services included in the MPC555 will be utilized by software.

In designing the actuation interface, versatility is sought so that the unit will be able to command any actuation technology including piezoelectrics, impact hammers and structural control devices, just to name a few. Therefore, to achieve an actuator-transparent interface, the interface will need to be designed so that it will be able to output a broad spectrum of voltage signals. At the core of the actuation interface is a digital-to-analog converter (DAC) that converts digital command signals generated by the MPC555 into analog representations acceptable for commanding actuators. The Texas Instruments DAC7624 is a 12-bit converter well suited for the task. The DAC7624 accepts 12-bit digital samples in parallel and outputs a zero-order-hold $(\mathrm{ZOH})$ analog signal from 0 to $2.5 \mathrm{~V}$ on four selectable channels. To attain the minimum settling time, only one channel of the quad-DAC is utilized in the actuation interface design. The settling time associated with each conversion is $10 \mu \mathrm{s}$, allowing digital data streams of up to $100 \mathrm{kHz}$. However, the DAC output range of $0-2.5 \mathrm{~V}$ is inadequate for commanding devices that require negative command signals. Therefore, the output signal of the DAC is fed to an instrumentation amplifier where the signal is range shifted and amplified. An Analog Devices AD620 amplifier is chosen to shift the DAC output to a zero mean (from $1.25 \mathrm{~V}$ ) and to amplify fourfold. This will result in an actuation interface capable of outputting command voltages from -5 to $+5 \mathrm{~V}$ using a 12-bit digital command signal issued by the MPC555. To assist the amplifier in range shifting the signal, the Analog Devices ADP667 voltage regulator is included in the actuation interface circuit. After the actuation interface is constructed in hardware, embedded firmware is written for the MPC555 to operate the interface with precise timing. To 
allow the DAC output sufficient time to settle, the maximum sample rate of the actuation interface is set at $100 \mathrm{kHz}$.

The wireless communication channel integrated with the wireless active sensing unit will immediately alleviate the burden of installing and maintaining cables in a structure. However, to be an effective substitute for cables, wireless radios chosen for integration with the wireless active sensing unit must be low-cost, far reaching and highly reliable. Previous wireless sensing unit designs have employed wireless modems such as the Proxim ProxLink and Proxim RangeLAN2 radio modems [7,16]. While both Proxim radios can propagate over $300 \mathrm{~m}$ in unobstructed open space, they consume over $150 \mathrm{~mA}$ of electrical current when transmitting and receiving data. To improve the power consumption characteristics of the wireless active sensing unit, a new wireless radio is considered for integration; in particular, the MaxStream 9XCite radio is chosen. The $9 \mathrm{XCite}$ communicates on the unregulated $900 \mathrm{MHz}$ radio band and can communicate with over-the-air data rates of $38.4 \mathrm{kbits}$ per second. The open space communication range of the radio is $300 \mathrm{~m}$ but is approximately $90 \mathrm{~m}$ when used in the interior of structures [17]. In contrast to the Proxim modems, the 9XCite radio only consumes $55 \mathrm{~mA}$ when transmitting data and $35 \mathrm{~mA}$ when receiving data. Use of the $9 \mathrm{XCite}$ radios reduce by at least one-third the power consumption demands of the Proxim radios without sacrificing openspace communication range. When the radios utilize the wireless channel, frequency hopping spread spectrum encoding is employed so that the radio channel is reliable and exhibits resilience to narrow-band interference.

After all of the electrical components have been selected, they are appropriately assembled to form a single prototype unit. To produce a prototype unit of the smallest form factor possible, the unit's functional modules (microcontroller, actuation interface and wireless modem) are housed on separate printed circuit boards that are stacked onto one another. While board stacking allows the unit form factor to be minimized, the approach will also render future hardware upgrades easier to complete. First, the Motorola MPC555 is purchased as a small starter development kit from Axiom (PB-555) where the microcontroller is mounted on a smallfootprint $(9 \times 9 \mathrm{~cm})$ printed circuit board. The output pins of the MPC555 are provided as header rows on the development kit board. The header rows of the Axiom board will be conveniently used to mount custom-made printed circuit boards on top. To house the actuation interface circuit and the external SRAM chip, a two-layer printed circuit board is designed and fabricated. This actuation interface/memory circuit board is design with dimensions close to those of the MPC555 board $(10 \times 10 \mathrm{~cm})$. The MaxStream wireless radios are purchased from the manufacturer on their own circuit board $(4 \times 7 \mathrm{~cm})$ and are placed as a third layer over the actuation interface circuit board. Figure 2 shows clearly the stack of printed circuit boards (three in total) assembled as a compact wireless active sensing unit. The final dimensions of the fully assembled wireless active sensing unit are $11 \times 10 \mathrm{~cm}$ in area and $4.5 \mathrm{~cm}$ in height. The cost of constructing the prototype, including the cost of the wireless modem, is approximately \$230. It should be noted that the final cost and size of the wireless active sensing unit are both limited by the resources available in the study's academic setting; industrial commercialization of the technology would reduce both cost and size by at least half. Table I summarizes the performance attributes of the proposed wireless active sensing unit prototype design.

Batteries represent the most probable power source for the wireless active sensing unit. However, wireless active sensing units embedded in infrastructure systems could reside in difficult-to-reach locations, making battery replacement a daunting task. Therefore, the power consumption characteristics of the wireless active sensing unit are of paramount concern. In 

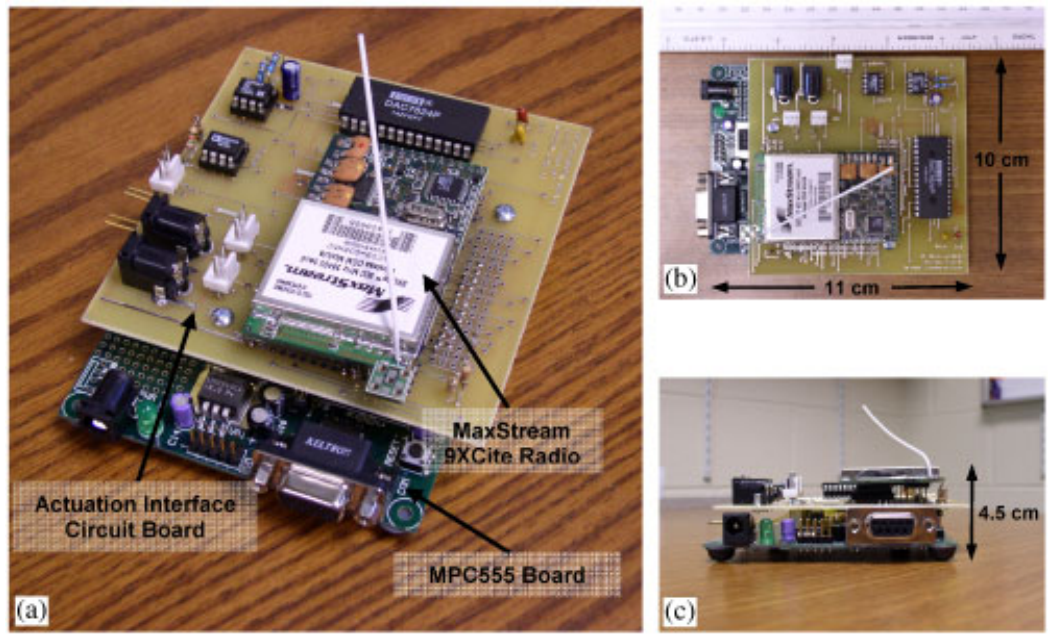

Figure 2. Fully constructed wireless active sensing unit: (a) perspective; (b) top; (c) side views.

Table I. Summary of the wireless active sensing unit hardware specifications.

\begin{tabular}{lll}
\hline Prototype subsystem & \multicolumn{1}{c}{ Attribute } & \multicolumn{1}{c}{ specification } \\
\hline Actuation interface & Signal type & Voltage \\
(1 channel) & Output range & -5 to $+5 \mathrm{~V}$ \\
& Resolution & $12-\mathrm{bits}$ \\
Sensing interface & Maximum sample rate & $100 \mathrm{kHz}$ \\
(32 channels) & Signal type & Voltage \\
& Input range & 0 to $+5 \mathrm{~V}$ \\
& Resolution & $10-\mathrm{bits}$ \\
Computational core & Maximum sample rate & $100 \mathrm{kHz}$ \\
(Motorola MPC555) & Clock speed & $40 \mathrm{MHz}$ \\
& Floating point & Hardware \\
Wireless communications & ROM (Internal) & $448 \mathrm{kbytes}$ \\
(Proxim 9XCite) & RAM (Internal/External) & $26 \mathrm{kbytes} / 512 \mathrm{kbytes}$ \\
& ISM frequency band & $900 \mathrm{MHz}$ \\
& Encoding & Frequency hopping \\
\hline
\end{tabular}

designing this prototype, unit functionality was of primary importance and designing the unit to be low-power, secondary. Future refinements on the current wireless active sensing unit design will emphasize the power consumption of the unit. From a usage standpoint, local data interrogation at the wireless sensor has been identified as a mechanism by which considerable battery power can be preserved [8]. Lithium-based batteries are considered for adoption with the wireless active sensing unit. Lithium batteries are superior to their alkaline counterparts because their output voltages remain constant over their operational lives and exhibit minimal deterioration when left unused for long periods of time. To assess the life expectancy of a lithium battery pack, the power demands of the individual components of the wireless active 
sensing unit are calculated. The entire wireless active sensing unit requires $170 \mathrm{~mA}$ of electrical current referenced at $5 \mathrm{~V}$ (which includes a conservative estimate of the electrical current required to drive piezoelectric actuation elements). Using a single battery pack consisting of four 3 V Panasonic BR-C polycarbon-monoflouride lithium batteries, each with a nominal capacity of $5000 \mathrm{mAh}$, the wireless active sensing unit would be capable of uninterrupted operation for approximately $30 \mathrm{~h}$ [18]. Naturally, duty cycle usage of the unit would be able to extend the life of the unit to one or two years before battery replacement would be necessary.

\section{EMBEDDED FIRMWARE FOR REAL-TIME DATA ACQUISITION}

Once the hardware is fully assembled into a wireless active sensing unit, software is required to manage the sensing and actuation interfaces, execute embedded engineering analyses and to exchange information with other units in the monitoring system's wireless network. The highlevel programming language $\mathrm{C}$ is chosen for writing software for the MPC555 microcontroller. To produce portable and high-quality code, the software is structured into three software modules (the sensing interface module, the actuation interface module, and the wireless channel module), each combining functions related to the same hardware subsystem. After compilation into binary machine code, the software is then burned into the MPC555 ROM memory banks for autonomous execution.

To meet the real-time demands of the sensing and actuation interfaces, both software modules must take advantage of the interrupt services provided by the MPC555 microcontroller. Interrupt services are a common feature included with microcontrollers to provide the opportunity to asynchronously interrupt software execution in order to service a subroutine that requires completion at a specific period in time. The sensing interface consists of the 10-bit analog-to-digital converter (ADC) that is contained in the MPC555 microcontroller's queued analog-to-digital converter module (QADC). The software module written to operate the sensing interface consists of functions that initialize the QADC, set the QADC clock and service the MPC555 interrupt signals when generated by the QADC, indicating the completion of an ADC conversion. Using the interrupt services, the ADC is read at a guaranteed time interval by the software module at sample rates as high as $100 \mathrm{kHz}$.

With the actuation interface constructed as a separate circuit external to the MPC555, realtime operation of the interface represents a more complex and challenging task. The Texas Instruments DAC7624 digital-to-analog converter (DAC) included in the actuation interface receives 12-bit digital data in parallel fashion (all bits presented at once). Additional control lines (CS and LDAC) are needed between the microcontroller and DAC that limit conversions to sample rates of $100 \mathrm{kHz}$ or less. To control the DAC conversion process, the general purpose input-output ports (MGPIO and MPWM) of the MPC555 microcontroller are employed. The 12-bit digital sample is output by the MGPIO port to the DAC7624 while the MPWM port is reserved for the control lines. The software module written to operate the actuation interface is essentially controlling the MGPIO and MPWM input-output ports of the MPC555. To establish a precise sample rate, the internal time processing unit (TPU) of the MPC555 microcontroller is used to generate a time signal tied to the interrupt services that will preempt software execution in accurately timed intervals. When the TPU interrupt is executed, an interrupt service routine loads data in the DAC and then initiates the conversion by bringing the CS and LDAC pins low. 
To validate the real-time performance of the actuation interface, the interface's analog output is connected to an Agilent 54621D mixed signal oscilloscope that will record the signal. Various waveforms are generated by the MPC555 and output by the actuation interface. First, a simple sine wave shown in Figure 3, is generated by the MPC555 at a low frequency of $1.35 \mathrm{~Hz}$. To generate the sine wave, a software math function is executed at each time-step to calculate the value of the sine function at that period in time. Execution of software to calculate the actuation output at each step is done to validate the ability to use the wireless active sensing unit in feedback control applications. The approach of generating a sine signal at each time step can consume significant amounts of processor time resulting in diminished performance at higher sine signal frequencies. Using the same approach, the sine signal frequency is increased to $160 \mathrm{~Hz}$ where the zero-order-hold $(\mathrm{ZOH})$ of the DAC output becomes well pronounced. The sample rate of the DAC in this case is set to a value that permits ample time for the sine function to calculate an output value. In the case of the $160 \mathrm{~Hz}$ sine signal, the DAC is commanded at $5.1 \mathrm{kHz}$. Clearly, higher frequency sine signals become increasingly difficult with $\mathrm{ZOH}$ effects dominating the signal output.

For applications where the output signal of the wireless active sensing unit is known a priori, an alternative approach to generating an output signal can be taken. To achieve high sample rates with minimal $\mathrm{ZOH}$ effects dominating the output signal, the desired output signal can be
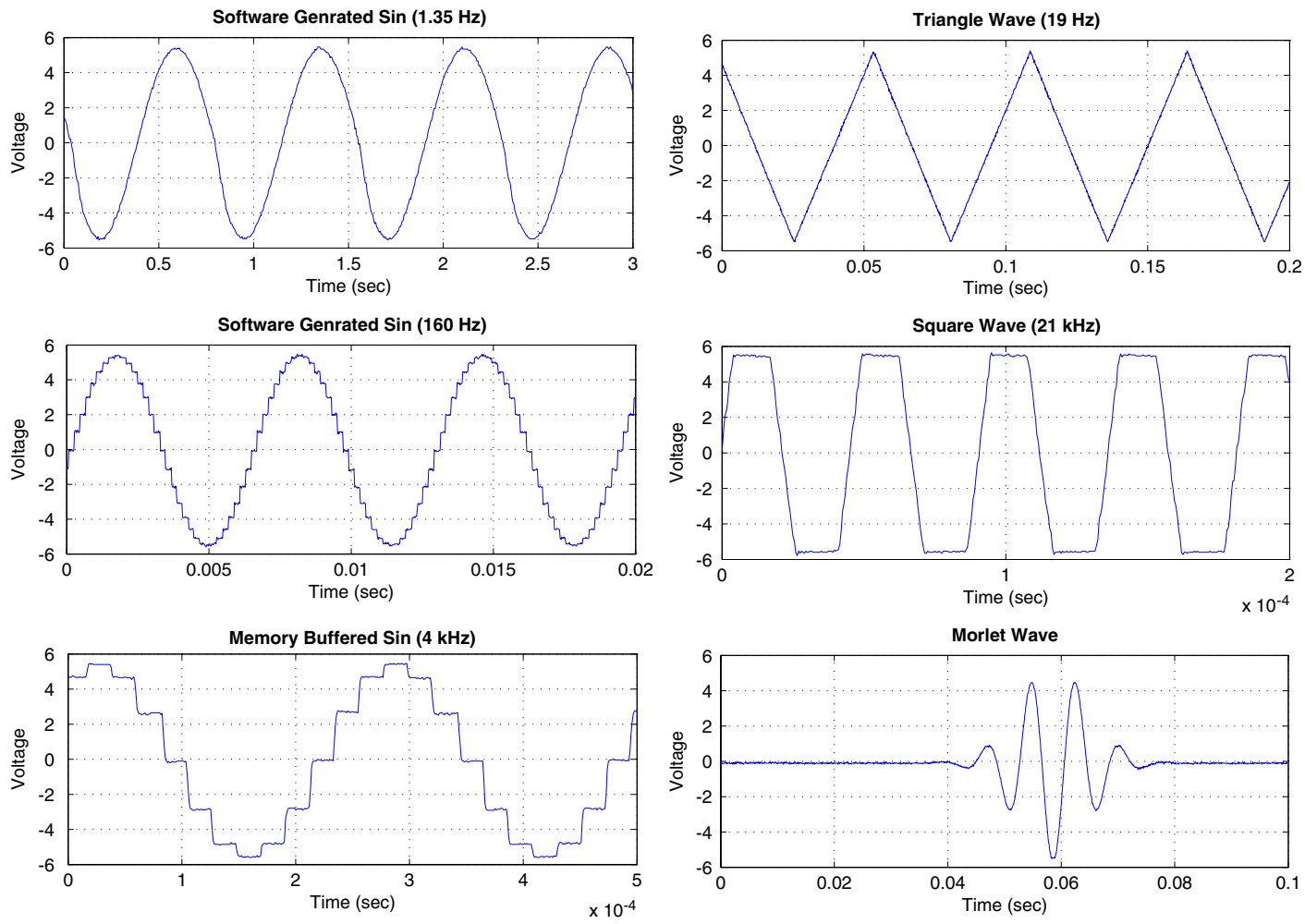

Figure 3. Waveforms generated by the wireless active sensing unit actuation interface. 
generated and stored in memory first. After the signal time-history has been stored, the signal can be executed by the actuation interface directly from memory. This approach eliminates the software overhead that would exist if the output has to be calculated. While the actuation interface can now be operated at higher sample rates, latencies in servicing MPC555 interrupts prevent the theoretical maximum sample rate of $100 \mathrm{kHz}$ from being achieved. Precision timing of the interface reveals the maximum permissible sample rate possible by using buffered memory for the output signal is $50 \mathrm{kHz}$. To validate the buffered memory approach, a $4 \mathrm{kHz}$ sine wave is generated at a sample rate of $50 \mathrm{kHz}$ and stored in memory prior to its output via the actuation interface. As shown in Figure 3, the high-rate sine signal is of relatively high quality, but $\mathrm{ZOH}$ effects dominate the signal.

To validate the ability of the actuation interface to generate linear signals, a $19 \mathrm{~Hz}$ triangle waveform is generated and output by the actuation interface. In a similar fashion, a highfrequency square waveform is generated to assess the output when held at a fixed voltage output. Some overshoot behavior is observed in the signal at the start of high and low regions. The signal also reveals some inherent white noise output by the DAC. In the last validation test, a Morlet waveform, widely used for generation of Lamb waves, is buffered in memory and output [13].

\section{PIEZOELECTRIC ACTUATED ALUMINUM PLATE EXPERIMENT}

The wireless active sensing unit coupled with active sensors could potentially be used to monitor a structure for the existence of crack damage. Using piezoelectric actuators mounted to the surface of metallic structural elements, Lamb waves can be generated for propagation through the structural element with corresponding element responses recorded. A number of researchers have investigated the use of Lamb waves and piezoelectric active sensors for the detection of structural damage. Sohn et al. [13] illustrate the use of piezoelectric actuators to generate Lamb waves in composite plates to identify delamination damage present in the plate. Taniguchi and Mita [19] have explored the application of pattern recognition and support vector machines (SVM) to screen a structure for damage based on the system's transient response to piezoelectric generated excitations.

To illustrate the role the proposed wireless active sensing unit could play in an automated structural health monitoring system, the prototype unit is used to identify crack damage in a structural element excited by active sensors. In particular, an aluminum plate to which piezoelectric pads have been surface mounted is intentionally cut to simulate crack damage. Using the wireless active sensing unit, the plate is excited with broadband acoustic excitations using one piezoelectric pad while a second pad is used to record the plate's corresponding strain response. For each set of input-output responses, autoregressive with exogenous input (ARX) time-series models are fit. Roots of the ARX model characteristic equation will be used to identify the occurrence and severity of structural damage within the aluminum plate.

\subsection{Aluminum plate experiment setup}

A cantilevered aluminum plate is chosen to serve as the structural element to which the piezoelectric actuators will be mounted. The plate is $0.3175 \mathrm{~cm}$ thick and has an area approximately $6.8 \times 28.6 \mathrm{~cm}$ in size. Piezoelectric actuators have been selected for excitation of 
the plate because their high-frequency operational spectrum can uniquely illustrate the performance of the wireless active sensing unit in high-speed sample rate applications. In this study, lead-zirconate-titanate (PZT) piezoelectric pads purchased from Piezo-systems, Inc. will be employed. A 0.191-mm-thick PZT sheet (T105-A4E-602) is cut to obtain small pads approximately $1.43 \times 1.43 \mathrm{~cm}$ in area. The PZT pads are nickel plated on both sides to render soldering of electrical connections easy. As shown in Figure 4, the piezoelectric pads are epoxy mounted to the top surface of the plate, $18.9 \mathrm{~cm}$ apart from each other. One pad is designated as the transmitting pad to be attached to the actuation interface of the wireless active sensing unit. The second pad is designated as the receiver and is connected to the unit's sensing interface. Based on commands issued by a remote computer via the wireless link, the wireless active sensing unit will excite the transmitting pad through the actuation interface with a variety of input signals pre-stored in memory. As the unit commands the transmitting pad, the unit will simultaneously collect the response of the plate, as measured by the second PZT pad, and store the input-output data in memory. Response data is subsequently transmitted to the remote computer by the wireless active sensing unit after the test is completed. To illuminate high-order response modes in the plate, both the sensing and actuation interfaces are operated at $40 \mathrm{kHz}$.

Numerous excitations can be considered for exciting the aluminum plate, but broadband white noise signals are chosen for this study. White noise signals are used because they are defined by constant power spectrums that will excite all of the aluminum plate modes that fall below the Nyquist frequency $(20 \mathrm{kHz})$. In total, a set of 10 white noise signals with varying levels of energy are stored in the wireless active sensing unit memory for repeated excitation of the plate. The white noise signals have standard deviations varying from 0.3 to $1.2 \mathrm{~V}$ in $0.1 \mathrm{~V}$ increments. All of the excitation signals are within the $\pm 5 \mathrm{~V}$ saturation limits of the actuation interface and draw little power from the wireless active sensing unit power supply. Figure 5 is the $1.0 \mathrm{~V}$ standard deviation white noise signal applied to the plate by the wireless active sensing unit and the corresponding plate response measured by the second PZT pad. Input-output timehistories of the other white noise excitations are similar to those presented in Figure 5.
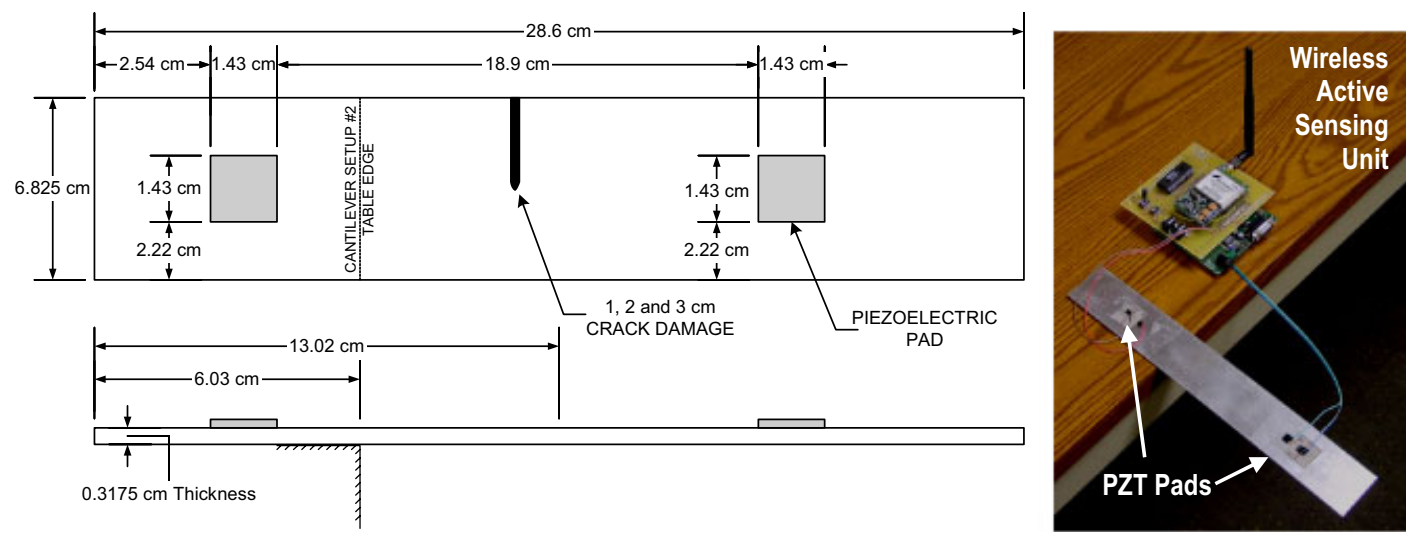

Figure 4. A cantilevered aluminum plate monitored by a wireless active sensing unit and PZT piezoelectric pads mounted to the plate surface. 

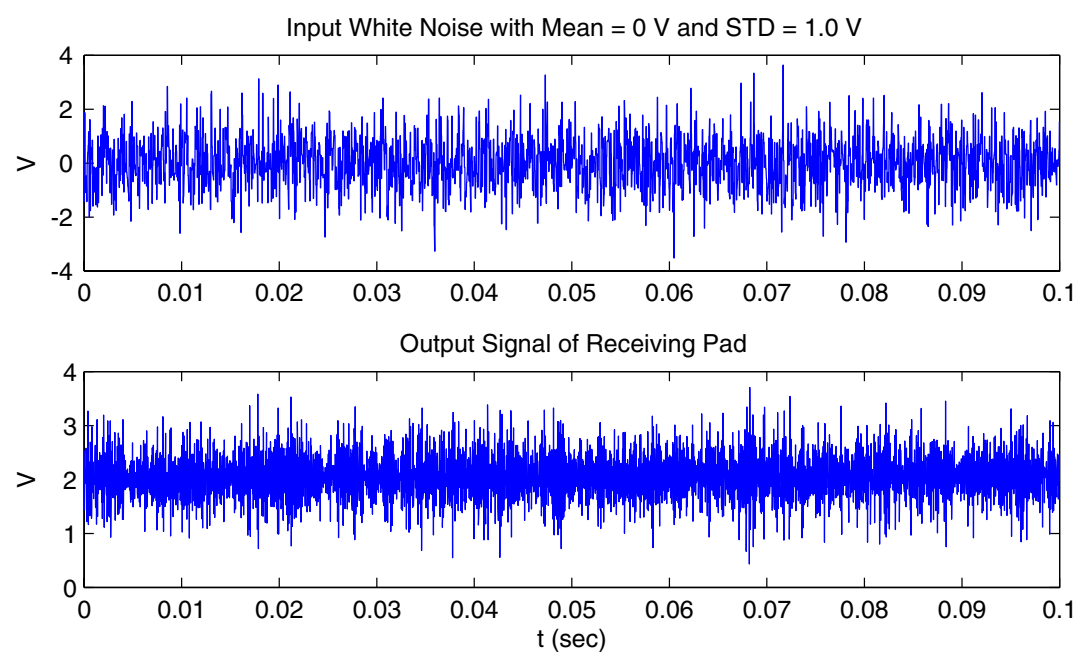

Figure 5. (Top) excitation signal (1.0 V STD) of the aluminum plate, and (bottom) corresponding plate response (sample rate $40 \mathrm{kHz}$ ).

\subsection{System identification of the aluminum plate system}

To provide a mathematical model for the aluminum plate behavior, system identification timeseries models can be formed from the plate's input-output time-history response data. From the large number of time-series models available, the ARX time-series model is selected for this study. ARX models are essentially linear difference equations that weigh past observations of the system output, $y$, with those of the system input, $u$, to make a prediction of the system output, $\hat{y}$, at time step $k$ :

$$
\hat{y}(k)=-a_{1} y(k-1)+\cdots-a_{n_{a}} y\left(k-n_{a}\right)+b_{1} u(k-1)+\cdots b_{n_{b}} u\left(k-n_{b}\right)
$$

The weights on the past observations of the system output are $a$, while those used for the input are $b$. The model assumes the system to be linear time-invariant and requires the input and output response data to both be stationary signals [20]. To accurately model the input-output response time-histories using an ARX model, the size of the ARX model needs to be chosen $a$ priori. When fitting ARX models to system response data, the size of the model will dictate its predictive qualities; generally, as sizes grow, model prediction errors will reduce. However, models too large are not necessarily better because they over-fit the measurement data and capture signal noise in addition to the system's physical behavior. To select a suitable size for the ARX models, two pairs of input-output time-history records are needed: the first is designated for calculation of the model and the second to verify the model prediction error. Using the first pair of input-output data, ARX model coefficients are calculated for various model sizes. Once the model is determined, its predictive error is calculated using the calculation as well as the validation data sets. The vector norm of the model's prediction error is a scalar quantity widely used to quantify the model's quality. When the model size first begins to increase, the norm of the prediction error of the ARX model using both sets of input-output data will rapidly decrease. This indicates the larger models are capturing the structural system's dynamics more 
completely. As the model size continues to grow, improvements in the model prediction error will occur at an increasingly slower rate. At one point, larger models will start to include signal noise; at this point, the prediction error of the second verification data set will begin to increase. As illustrated in Figure 6, an acceptable range of model orders are those that fall between the point where the calculation data set error reduces at a slow rate and the point where the prediction error of the verification data set begins to increase. For the aluminum plate, the $0.3 \mathrm{~V}$ excitation input-output response histories are chosen as the calculation data set for the ARX models and the $0.4 \mathrm{~V}$ input-output response time-histories are selected as the verification data set. The selection of these two input-output data sets was arbitrary. Using these two data sets, the optimal number of weights on the ARX model inputs and outputs, denoted $n_{a}$ and $n_{b}$ respectively, is determined to be $n_{a}=21$ and $n_{b}=4$.

Once the appropriate ARX model order has been established, ARX time-series models can be calculated from the input-output response history of the aluminum plate system. A leastsquares estimation approach is taken to find the optimal ARX coefficients by minimizing a quadratic cost function of the model error time-history, $e(k)=\hat{y}(k)-y(k)$. To employ a leastsquares solution for finding the optimal ARX model coefficients, the input-output response of the plate is assembled into a matrix form with the ARX model difference equation written for each output observation of the system:

$y=\left\{\begin{array}{c}y\left(n_{a}+1\right) \\ y\left(n_{a}+2\right) \\ \vdots \\ y(N)\end{array}\right\}=\left[\begin{array}{cccccc}y\left(n_{a}\right) & \cdots & y(1) & u\left(n_{a}\right) & \cdots & u\left(n_{a}-n_{b}+1\right) \\ y\left(n_{a}+1\right) & \cdots & y(2) & u\left(n_{a}+1\right) & \cdots & u\left(n_{a}-n_{b}+2\right) \\ \vdots & \ddots & \vdots & \vdots & \ddots & \vdots \\ y(N-1) & \cdots & y\left(N-n_{a}\right) & u(N-1) & \cdots & u\left(N-n_{b}\right)\end{array}\right]\left(\begin{array}{c}-a_{1} \\ \vdots \\ \vdots \\ -a_{n_{a}} \\ b_{1} \\ b_{n_{b}}\end{array}\right\}=A c$

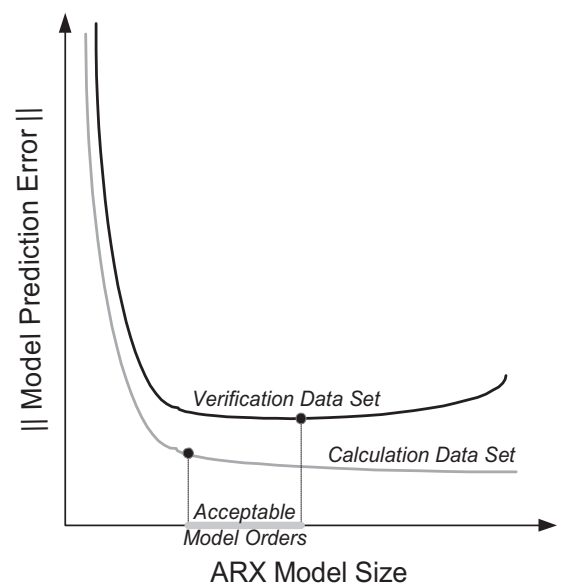

Figure 6. Behavior of the ARX prediction error as a function of the model size. 
It is generally assumed that more response samples are collected than the number of coefficients used in the ARX model $\left(N>>n_{b}+n_{a}\right)$. In this case, the measurement matrix, $A$, will be over-determined and full rank. To find the vector of ARX model coefficients, $c$, the leastsquares solution of Equation (2) is given by:

$$
c=\left(A^{T} A\right)^{-1} A^{T} y
$$

After an ARX model is formed for the plate system in the time domain, the model is transferred to the frequency domain. Provided the system is discretely sampled in time, the $Z$-transform is used to derive the system transfer function in the complex $Z$-domain:

$$
H(z)=\frac{Y(z)}{U(z)}=\frac{b_{1} z^{-1}+\cdots b_{4} z^{-4}}{1+a_{1} z^{-1}+\cdots a_{21} z^{-21}}
$$

The motivation for converting the ARX model to the complex frequency domain is to obtain from the resulting transfer function, the frequencies and damping ratios of the system's response modes. In particular, the denominator of the transfer function is the polynomial characteristic equation of the linear system. An interesting feature of the characteristic equation is that the complex roots of the equation, also termed poles, embody the frequency $\omega$ and damping ratio $\xi$ of each uncoupled response mode (where $T$ is the time-step of the sampled system):

$$
z_{\text {ROOT }}=e^{\left(-\xi \omega_{n} \pm \omega_{n} \sqrt{1-\xi^{2}} j\right) T}
$$

The poles of the system transfer function can be drawn upon the real-imaginary complex plane. Poles within the unit circle are denoted as stable, while those outside the unit circle represent unstable modes of the system. The complex plane is an incredibly powerful visualization tool widely used in the design of feedback control systems. Upon the complex plane, contours of constant frequency and damping can be superimposed with the plotted poles to provide visual reference of pole frequency and damping values. For this reason, the complex plane will be used in this study as an expressive domain to analyze if a structure should be characterized as damaged or undamaged.

\subsection{Identification of crack damage in the aluminum plate}

To simulate cracking of the aluminum plate, a hacksaw is used to introduce a cut between the PZT pads that is orthogonal to the plate's longitudinal axis, as shown in Figure 4. The plate is cut in multiple steps, resulting in simulated cracks of various lengths. In total, three cuts are incrementally made: 1,2 and $3 \mathrm{~cm}$ long. The 10 white noise excitations stored in the wireless active sensing unit memory bank are identically applied to the aluminum plate when it is undamaged and when it is cut. In total, 10 ARX models are fit to the input-output response pairs for the plate in each structural state. Using the calculated ARX model coefficients, the poles of the ARX model transfer function are determined. For each ARX model, a total of 21 poles are determined with the first pole a real number and the remaining 20 poles as 10 conjugate pole pairs. It is hypothesized that, as damage is introduced in the aluminum plate, the stiffness and damping properties of the plate change, resulting in a detectable change in the location of poles upon the complex plane. Plotted in Figure 7 are the ARX model poles corresponding to the undamaged aluminum plate. Superimposed on the complex plane with dotted lines are contours of constant damping originating from $z=1$ and contours of constant frequency originating from the unit circle boundary. When the poles are plotted for the 10 


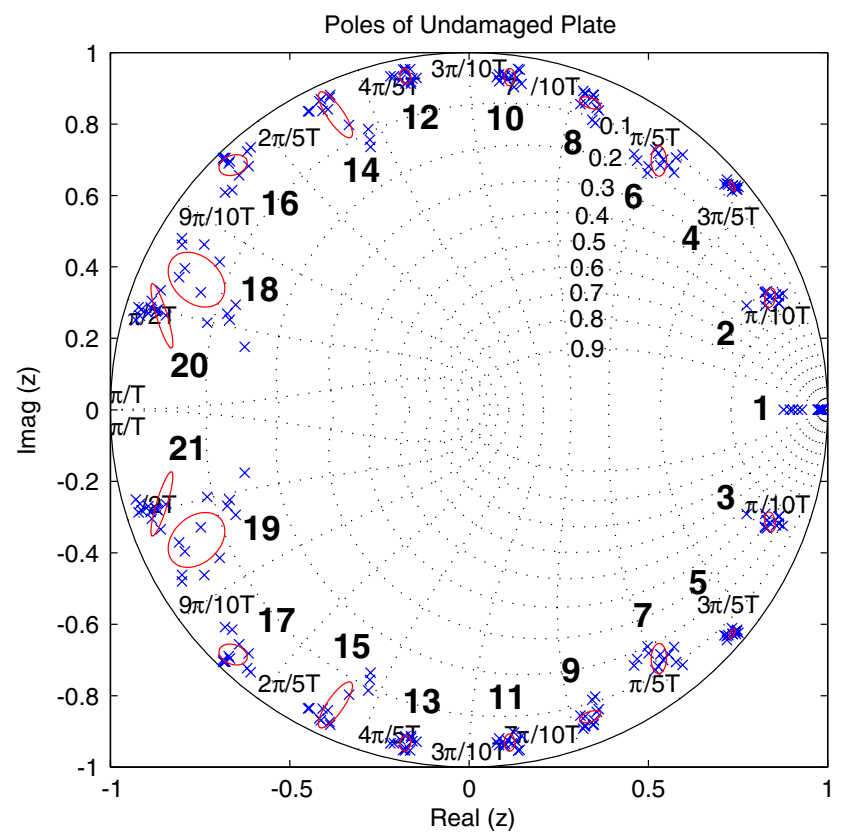

Figure 7. Location of ARX model pole clusters upon the complex $Z$-plane (with each pole cluster numbered 1-21).

excitation sources, the poles naturally form 21 clusters with variability of the poles evident for each cluster. The variability in pole location can be attributed to low levels of electrical noise within the wireless active sensing unit actuation and sensing interfaces. Bivariate normal probability density functions are estimated for each pole cluster of the plate in the undamaged and damaged states. Superimposed with each pole cluster in Figure 7 is an elliptical level curve representing the first standard deviation of the estimated normal density function.

The location of ARX pole clusters exhibit sensitivity to cracks introduced in the aluminum plate. Furthermore, as the crack in the aluminum plate grows, some of the ARX model pole clusters migrate on the complex plane in concert with the cut length. The normal probability density functions for each pole cluster are tracked upon the complex plane as the plate is incrementally damaged. While all of the clusters exhibit some migration, it is observed that pole clusters 2, 6 and 12 exhibit the greatest sensitivity to the growing cut. Figure 8 presents the migration of the estimated normal density function for each of these damage sensitive pole clusters. For all three clusters, a significant change in the cluster location is experienced when damage is first introduced in the plate. For the $1-\mathrm{cm}$-long cut, the mean of the pole cluster is outside of the level curve corresponding to the first standard deviation of the normal density function of the undamaged plate; clearly, this is a statistically significant migration. As damage is increased, the means of the pole clusters move an increasing distance away from the corresponding undamaged plate's clusters. The absolute difference between the estimated normal density function mean of the damaged and undamaged plate is measured for each cut length. Also presented in Figure 8 for clusters 2, 6 and 12, are plots of the norm of the complex 

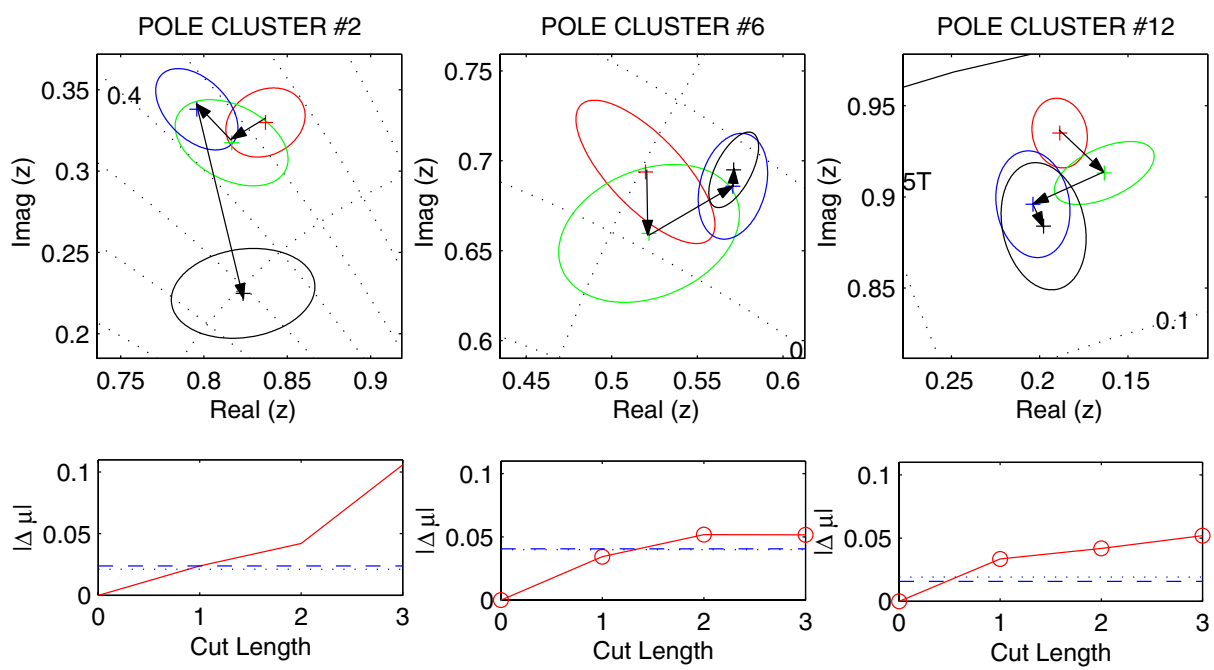

Figure 8. Migration patterns of the 2nd, 6th, and 12th ARX model pole cluster as a function of damage (arrow indicates migration from no damage to a 1,2 and $3 \mathrm{~cm}$ crack).

change in cluster mean for each cut length; clearly, the cluster means increase in tandem with the growing cut length. Superimposed upon the same plots are dashed and dotted lines that represent the standard deviation of the estimated normal density function in the real and imaginary coordinates, $\sigma_{R e}$ and $\sigma_{I m}$, for the undamaged plate clusters. The intention of superimposing these standard deviations is to provide a benchmark for assessing the statistical significance of the pole cluster migrations. For the 1 -cm-long cut, the absolute mean difference for cluster 2 and 6 is approximately equal to the standard deviations of the estimated normal density function of the undamaged plate. However, as the cut lengthens, the migration of the cluster becomes increasingly significant as shown. As a result of symmetry in the complex plane, clusters 3, 7 and 13 behave in identical manner to clusters 2, 6 and 12 respectively. The other seven remaining pole clusters exhibit only minor sensitivity to the introduction of crack damage with clusters migrating within the first standard deviation of the cluster for the undamaged plate.

The method proposed for identifying crack damage in the aluminum plate shares many similarities with damage detection methods previously proposed that considered shifts in the natural frequency of the dynamic system modes. This method is potentially more powerful because system poles contain information on both modal natural frequencies and damping ratios. For many of the cut lengths, the additional damage resulted in pole cluster migrations consistent with changes in frequency, damping, or a combination of the two. Had only frequency been considered, many of the pole migrations corresponding in changes in the damping would not have been detected.

There exists a strong relationship between the modal frequencies calculated from frequency response functions and the poles on the complex plane. The transfer function of Equation (4) can be determined for every location on the complex plane, except at the pole locations where $H(z)$ is infinite. Hence, the transfer function describes a three-dimensional contour above the 
complex plane. If this transfer function contour is sliced along the unit circle, the discrete frequency response function would be derived. Hence, peaks that correspond to modal frequencies in the frequency response functions are really the result of poles in the complex plane pulling the transfer function contour towards infinity. In essence, the discrete frequency response function is a two-dimensional subset of the more complex three-dimensional transfer function contour.

\section{CONCLUSIONS}

To reduce installation costs and enhance functionality, innovative technologies are currently being adopted in structural monitoring systems. This study has explored an extension of the wireless structural monitoring paradigm by tightly integrating with a wireless sensing unit, the ability to actuate. Providing a means for direct interaction with the physical system in which it is installed, wireless active sensing units can play a major role in structural health monitoring systems. The design of an actuation interface ensures the unit is capable of commanding active sensors, impact hammers and large-scale actuators. After selecting optimal hardware components, the components are assembled into a compact wireless active sensing unit whose volume is less than $500 \mathrm{~cm}^{3}$ and whose cost is approximately $\$ 230$. Various validation studies are performed to assess the capabilities of the actuation interface. The result of these waveform output tests revealed excellent outputs defined by low noise and high accuracy. However, the tests reveal code latencies that reduce the effective maximum sample rate of the actuation interface to $50 \mathrm{kHz}$.

To illustrate the role the prototype wireless active sensing unit might play in future structural health monitoring systems, a simple laboratory experiment is devised for illustration. An aluminum plate, with two PZT piezoelectric pads epoxy mounted to its surface, is monitored by the wireless active sensing unit for signs of damage. The study proposes a novel interrogation approach for detecting crack damage in metallic structural elements. Using the characteristic equation roots of ARX models fit to the input-output response data, damage can be successfully identified in the aluminum plate. In particular, a set of broadband excitations are applied to the plate in a given structural state (undamaged versus damaged) so that the distribution of ARX model poles upon the complex plane can be statistically described using estimated bivariate normal probability density functions. As damage is introduced into the aluminum plate, an observable change the location of pole clusters upon the complex plane is witnessed. The migration of the pole clusters were shown to be statistically significant by comparing the absolute difference of cluster means with the standard deviations of the undamaged plate's estimated normal probability density functions. Hence, the cluster mean appears to be a promising indicator of the existence of crack damage with the degree of cluster migration strongly correlated with the length of the crack in the aluminum plate.

Future work will explore the improvement of the actuation interface of the wireless active sensing unit design. Higher sample rates, in excess of $1 \mathrm{MHz}$, are sought for selective excitation of desired symmetric and antisymmetric plate modes using Lamb waves. The energy consumption characteristics of the total unit can be further reduced by adopting microcontrollers that consume less power. The paper described a viable approach to identifying crack damage in structural elements. With the approach proven to identify damage, firmware is currently being written to allow the wireless active sensing unit to autonomously execute the 
methodology. The pole migration method proposed in this study was only intended to assist in illustrating the capabilities of the prototype design. Clearly, many other damage detection methods that take advantage of an ability to actuate the structural system can also be considered. Aside from damage detection, the wireless active sensing unit can be used in structural control systems. Research efforts are already underway exploring the feasibility of the technology for a number of control applications.

\section{ACKNOWLEDGEMENTS}

The author would like to express his gratitude to Arvind Sundararajan and Professor Kincho Law of Stanford University and Dr Hoon Sohn and Dr Chuck Farrar of Los Alamos National Laboratory for providing assistance in the design and fabrication of the wireless active sensing unit prototype. This research is partially funded by the National Science Foundation under grant numbers CMS-9988909 (Stanford University) and CMS-0421180 (University of Michigan). Additional support was provided by Los Alamos National Laboratory (contract number 75067-001-03) and the College of Engineering, University of Michigan.

\section{REFERENCES}

1. Chase S, Ghasemi H. A vision for highway bridges for the 21st century. Proceedings of the 4th International Workshop on Structural Health Monitoring, Stanford, CA, 15-17 September, 2003; 205-213.

2. International Conference of Building Officials (ICBO). 2001 California building code-California code of regulations. Title 24, Part 2, Volume 2, 2002.

3. Doebling SW, Farrar CR, Prime MB, Shevitz DW. Damage Identification and Health Monitoring of Structural and Mechanical Systems from Changes in their Vibration Characteristics: a Literature Review. Report No. LA-13070-MS, Los Alamos National Laboratory. Los Alamos, NM, 1996.

4. Celebi M. Seismic Instrumentation of Buildings (with Emphasis on Federal Buildings). Report No. 0-7460-68170, United States Geological Survey, Menlo Park, CA, 2002.

5. Moore GE. Cramming more components onto integrated circuits. Proceedings of the IEEE 1968; 86(1):82-85.

6. Straser EG, Kiremidjian AS. A modular, wireless damage monitoring system for structures. John A. Blume Center Technical Report No. 128, Stanford University, Stanford, CA, 1998.

7. Lynch JP, Law KH, Kiremidjian AS, Carryer E, Farrar CR, Sohn H, Allen DW, Nadler B, Wait JR. Design and performance validation of a wireless sensing unit for structural monitoring applications. Structural Engineering and Mechanics 2004; 17(3-4):393-408.

8. Lynch JP, Sundararajan A, Law KH, Kiremidjian AS, Carryer E. Embedding damage detection algorithms in a wireless sensing unit for operational power efficiency. Smart Materials and Structures 2004; 13(4):800-810.

9. Arici Y, Mosalam KM. Modal analysis of a densely instrumented building using strong motion data. Proceedings of the International Conference on Applications of Statistics and Probability in Civil Engineering (ICASP 2003), San Francisco, CA, 6-9 July, 2003; 419-426.

10. Glaser SD. Some real-world application of wireless sensor nodes. Proceedings of Sensors and Smart Structure Technologies for Civil, Mechanical and Aerospace Systems, SPIE, San Diego, CA, 14-18 March, 2004; 5391:344-355.

11. Alleyne DN, Cawley P. The interaction of lamb waves with defects. IEEE Transactions on Ultrasonics, Ferrorelectrics and Frequency Control 1992; 39(3):381-397.

12. Hurlebaus S. A contribution to structural health monitoring using elastic waves. PhD Thesis, University of Stuttgart, Stuttgart, Germany, 2002.

13. Sohn H, Park G, Wait JR, Limback NP, Farrar CR. Wavelet-based active sensing for delamination detection in composite structures. Smart Materials and Structures 2004; 13(1):153-160.

14. Tua PS, Quek ST, Wang Q. Detection of cracks in plates using piezo-actuated Lamb waves. Smart Materials and Structures 2004; 13(4):643-660.

15. Park G, Cudney HH, Inman DJ. Impedance-based health monitoring of civil structural components. Journal of Infrastructure Systems 2000; 6(4):152-160.

16. Lynch JP, Law KH, Kiremidjian AS, Kenny TW, Carryer E. A wireless modular monitoring system for civil structures. Proceedings of the International Modal Analysis Conference (IMAC XX), Los Angeles, CA, 4-7 February, $2002 ; 1-6$ 
17. MaxStream, Inc. 9 XCite advanced programming and configuration: advanced manual. MaxStream, Inc.: Lindon, UT, 2003.

18. Panasonic, Inc. Poly-carbonmonoflouride lithium batteries: individual specifications BR-C. Panasonic, Inc.: Secaucus, NJ, 2003.

19. Taniguchi R, Mita A. Support Vector Machine based active damage detection method. Proceedings of the 4th International Workshop on Structural Health Monitoring, Stanford, CA, 15-17 September, 2003; 749-756.

20. Ljung L. System Identification-Theory for the User. Prentice-Hall: New Jersey, 1999. 